\title{
Traveling Baseball Players' Problem in Korea
}

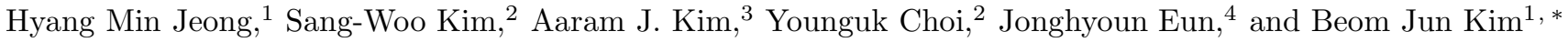 \\ ${ }^{1}$ Department of Physics and BK21 Physics Research Division, \\ Sungkyunkwan University, Suwon 440-746, Korea \\ ${ }^{2}$ Department of Physics, Soongsil University, Seoul 156-743, Korea \\ ${ }^{3}$ Department of Physics and Astronomy and Center for Theoretical Physics, \\ Seoul National University, Seoul 151-747, Korea \\ ${ }^{4}$ Department of Physics and Astronomy, University of California Los Angeles, Los Angeles, California 90095-1547, USA
}

(Dated: September 3, 2018)

\begin{abstract}
We study the so-called the traveling tournament problem (TTP), to find an optimal tournament schedule. Differently from the original TTP, in which the total travel distance of all the participants is the objective function to minimize, we instead seek to maximize the fairness of the round robin tournament schedule of the Korean Baseball League. The standard deviation of the travel distances of teams is defined as the energy function, and the Metropolis Monte-Carlo method combined with the simulated annealing technique is applied to find the ground state configuration. The resulting tournament schedule is found to satisfy all the constraint rules set by the Korean Baseball Organization, but with drastically increased fairness in traveling distances.
\end{abstract}

PACS numbers: 89.65.-s, $89.75 . \mathrm{Fb}$

Keywords: Monte-Carlo simulation, optimization, traveling salesman problem, traveling tournament problem, sports tournament, baseball league

\section{INTRODUCTION}

The traveling salesman problem (TSP) is the one of the most important optimization problems in computational sciences [1]. In TSP, for given number of places to visit, a traveling salesman hopes to minimize the total travel distance within the constraint that she is allowed to visit each place only once. Hinted by the similarity to the problem of finding the ground state of a system with a rugged energy landscape, TSP has also been broadly studied in physics community 2, 3]. There are also biologically motivated interesting methods in the study of TSP, such as the genetic algorithm [4] and the ant colony optimization [5, 6]. Although direct formulations based on TSP have broad range of applicability, we note that there are closely-related, but different, problems in reality. One among such problems is the scheduling of games in sports: In contrast to TSP, which is unilateral, i.e., the traveling salesman is not required to take into account existences of other salesmen, many sports games are bilateral, i.e., two teams or players should meet somewhere to play a game. Designing of a tournament schedule in sports league is notoriously difficult problem to solve, which has been studied mostly in the area of computational science [7, 8]. Recently, there has been a significant development in the traveling tournament problem (TTP) [9], in which the task is to construct the optimal timetable to minimize the total travel distance under the constraint of a given home-and-away pattern. Not surprisingly, the simulated-annealing method [10], and the ant colony optimization method [1] have been applied,

\footnotetext{
* Corresponding author: beomjun@skku.edu
}

TABLE I. Korean Baseball League of eight teams. Note that two teams (Doosan and LG) share the same home stadium (Jamsil). The latitude and the longitude are for the locations of stadiums. Acronyms used in the present work are also listed.

\begin{tabular}{c|c|c|c}
\hline \hline$\#$ & team name & stadium & (latitude, longitude) \\
\hline 1 & Samsung Lions (SS) & Daegu & $(35.882,128.585)$ \\
2 & SK Wyverns (SK) & Munhak & $(37.436,126.689)$ \\
3 & Lotte Giants (LT) & Sajik & $(35.195,129.059)$ \\
4 & Kia Tigers (KA) & Gwangju & $(35.168,126.888)$ \\
5 & Doosan Bears (DS) & Jamsil & $(37.514,127.075)$ \\
6 & LG Twins (LG) & Jamsil & $(37.514,127.075)$ \\
7 & Hanwha Eagles (HH) & Daejeon & $(36.314,127.428)$ \\
8 & Nexen Heroes (NX) & Mokdong & $(37.531,126.881)$ \\
\hline \hline
\end{tabular}

as well as more conventional methods in computer science [12], to tackle TTP and similar problems.

In this work, we study the TTP, but with an important alteration: Instead of minimizing the total travel distance, we minimize the unfairness of the travel distance. This, we believe, is even more important than just minimizing the total travel distance summed over all the teams in the sports league. If one team should travel much longer distance than others, the team has less chance to win the tournament. Consequently, for the sake of fairness, the difference of the travel distance of each team must be made as small as possible, even though it might sacrifice the total sum of the travel distances of all the teams. In this respect, the present work is related with discussions of social justice in which equality of opportunity is the one of the key issues [13]. Specifically, this work is motivated by the common belief among baseball fans in Korea. One team (Lotte Giants) is located 


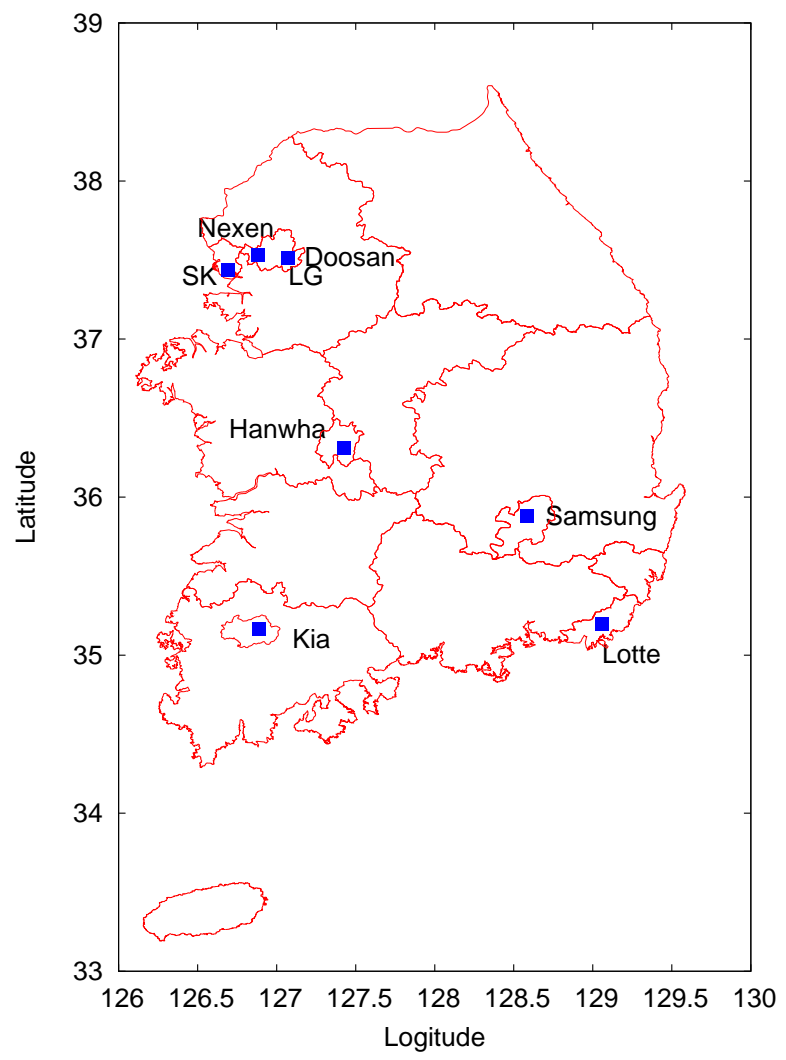

FIG. 1. (Color online) Locations of 8 baseball teams in Korean Baseball League. Two teams, Doosan Bears and LG Twins, share the same home stadium. Lotte is located at the farthest distance from Seoul.

in the most southern part of Korean peninsula, and thus it is not fair for the team to compete with all others on the same ground. Interestingly, this claim, mostly by fans of Lotte Giants - they are also well known to be very enthusiastic by the way - has some truth in it, and the Korean Baseball Organization (KBO) set up a rule trying to reduce the travel distance of the Lotte Giants: KBO allows the team to have more away games in a row without returning to their home (see Sec. II).

The present paper is organized as follows: In Sec. II], we describe in detail the current scheduling scheme of KBO, which is followed by the formulation of the problem in terms of the Monte-Carlo method in statistical physics in Sec. III. The results are presented in Sec. IV] which are summarized in Sec. $\mathrm{V}$ with some discussion and proposal.

\section{SCHEDULING OF KOREAN BASEBALL LEAGUE}

In Korean Baseball League (KBL), there are now eight teams as shown in Table I. Two teams (5 and 6, Doosan Bears and LG Twins) based on Seoul, the capital of South Korea with over 10 million population, share the same stadium Jamsil. The locations of home stadiums are denoted in the form of (latitude, longitude) in Table I] We also display the home positions of baseball teams on the map of South Korea in Fig. 1, which clearly shows that teams are not uniformly scattered across the country and that there are more teams around Seoul area. Since baseball teams usually travel by bus (in Monday afternoon and Thursday night), we measure the distance for a given pair of two teams by the output from the path finding service provided by a company in Internet. More specifically, we type in the location of the stadium of the team $i$ as the departure position, and then that of the team $j$ as the destination position. The Web service provided by the company Naver (http://www.naver.com) then returns us the distance $d_{i j}$ and the estimated time $t_{i j}$ for the travel path from $i$ to $j$, as shown in Tables II and III respectively. It is to be noted that $d_{i j} \neq d_{j i}$, and $t_{i j} \neq t_{j i}$, although the differences are not so substantial. Figure 2 displays (a) $\bar{d}_{i} \equiv(1 / 16) \sum_{j=1}^{8}\left(d_{i j}+d_{j i}\right)$ and (b) $\overline{t_{i}} \equiv(1 / 16) \sum_{j=1}^{8}\left(t_{i j}+t_{j i}\right)$, respectively. We note that Lotte, Kia, and Samsung have larger average distances and times than other teams. The shorter average distances for Doosan, LG, SK, and Nexen are because of their locations near Seoul, and Hanwha has a short average distance because it is located in the middle of the country. From Fig. 2, we recognize that if the tournament scheduling is made without any consideration of the inequality of the average distances, some teams must travel longer distances spending longer times in traffic than other teams, and thus those teams might have less chance to win the games. The original formulation of TTP was made to minimize the total travel distances of all teams. However, in view of the unequal distance distribution in KBL (see Fig. 2), it is natural to shift the optimization focus toward the fairness of the tournament schedule, which will be the main research theme of the present work.

We next describe the tournament scheduling scheme of KBO in detail. At the time of writing of the present paper (year 2012), each team in one season plays 133 games, which corresponds to $19(=133 / 7)$ games for each pair of two teams, making the total number of games 532 $(=133 \cdot 8 / 2)$. The scheduling constraints set by KBO are as follows:

1. The number of home/away games are either $66 / 67$ or $67 / 66$ (with $66+67=133$ ), respectively. If a team had 66 (67) home games in the last season, 67 (66) home games are assigned. In 2011, Kia, LG, Hanwha, and Nexen had 67 home games and Samsung, SK, Lotte, and Doosan had 66 home games. In 2012, therefore, the four former teams should have 66 home games and the latter 67 home games.

2. There is no game on Monday. From Tuesday to Thursday two teams play three games in a row at the same stadium (home stadium of one of the two teams), and then move to other stadium to play three more games in a row from Friday to Sunday. 
TABLE II. Distance matrix for baseball teams. The number at the $i$ th row and $j$ th column denotes the distance $d_{i j}$ from $i$ to $j$ in units of $\mathrm{km}$, and the average distances $\bar{d}_{i \bullet}$ and $\bar{d}_{\bullet j}$ are defined by $\bar{d}_{i \bullet} \equiv(1 / 8) \sum_{j=1}^{8} d_{i j}$ and $\bar{d}_{\bullet j} \equiv(1 / 8) \sum_{i=1}^{8} d_{i j}$. For the team index $i$, see Table 1

\begin{tabular}{c|c|c|c|c|c|c|c|c}
\hline \hline from $\backslash$ to & $1(\mathrm{SS})$ & $2(\mathrm{SK})$ & $3(\mathrm{LT})$ & $4(\mathrm{KA})$ & $5,6(\mathrm{DS}, \mathrm{LG})$ & $7(\mathrm{HH})$ & $8(\mathrm{NX})$ & $\bar{d}_{i \bullet}$ \\
\hline $1(\mathrm{SS})$ & 0 & 299.0 & 106.7 & 220.0 & 279.2 & 147.4 & 298.2 & 203.7 \\
\hline $2(\mathrm{SK})$ & 298.6 & 0 & 404.8 & 315.3 & 54.6 & 178.5 & 30.9 & 167.1 \\
\hline $3(\mathrm{LT})$ & 105.7 & 404.9 & 0 & 254.9 & 379.6 & 253.1 & 404.1 & 272.7 \\
\hline $4(\mathrm{KA})$ & 215.6 & 309.5 & 253.9 & 0 & 294.2 & 174.7 & 308.6 & 231.3 \\
\hline $5,6(\mathrm{DS}, \mathrm{LG})$ & 278.8 & 53.1 & 385.1 & 290.5 & 0 & 162.6 & 23.2 & 149.16 \\
\hline $7(\mathrm{HH})$ & 147.3 & 181.9 & 253.6 & 175.4 & 174.9 & 0 & 181.2 & 159.2 \\
\hline $8(\mathrm{NX})$ & 301.9 & 29.4 & 408.1 & 312.7 & 22.3 & 184.8 & 0 & 180.0 \\
\hline $\bar{d}_{\bullet} j$ & 203.4 & 166.4 & 274.7 & 232.4 & 150.6 & 158.0 & 158.7 & \\
\hline \hline
\end{tabular}

TABLE III. Time matrix for baseball teams. The number at the $i$ th row and $j$ th column denotes the travel time $t_{i j}$ from $i$ to $j$ in units of min, and the average traveling times $\bar{t}_{i \bullet}$ and $\bar{t}_{\bullet j}$ are defined by $\bar{t}_{i \bullet} \equiv(1 / 8) \sum_{j=1}^{8} t_{i j}$ and $\bar{t}_{\bullet j} \equiv(1 / 8) \sum_{i=1}^{8} t_{i j}$.

\begin{tabular}{c|c|c|c|c|c|c|c|c}
\hline \hline from $\backslash$ to & $1(\mathrm{SS})$ & $2(\mathrm{SK})$ & $3(\mathrm{LT})$ & $4(\mathrm{KA})$ & $5,6(\mathrm{DS}, \mathrm{LG})$ & $7(\mathrm{HH})$ & $8(\mathrm{NX})$ & $\bar{t}_{i \bullet}$ \\
\hline $1(\mathrm{SS})$ & 0 & 239 & 105 & 222 & 215 & 139 & 242 & 172 \\
\hline $2(\mathrm{SK})$ & 233 & 0 & 310 & 234 & 77 & 161 & 49 & 143 \\
\hline $3(\mathrm{LT})$ & 113 & 325 & 0 & 208 & 307 & 221 & 336 & 227 \\
\hline $4(\mathrm{KA})$ & 217 & 255 & 206 & 0 & 239 & 149 & 271 & 197 \\
\hline $5,6(\mathrm{DS}, \mathrm{LG})$ & 211 & 74 & 288 & 230 & 0 & 148 & 39 & 124 \\
\hline $7(\mathrm{HH})$ & 129 & 180 & 209 & 151 & 161 & 0 & 189 & 148 \\
\hline $8(\mathrm{NX})$ & 231 & 47 & 311 & 229 & 38 & 162 & 0 & 132 \\
\hline $\bar{t}_{\bullet} j$ & 168 & 149 & 215 & 188 & 130 & 141 & 146 & \\
\hline \hline
\end{tabular}

3. The first week of the season is an exception: New season starts with an opening ceremony on Saturday. Each team plays two games on Saturday and Sunday without changing counterpart. The four stadiums of the first week are the homes of the top four teams of the season 2010 (SK, Samsung, Doosan, and Lotte). The final rank in 2010 determines the counterpart of each game [rank 1 (SK) plays with rank 5 (Kia), 2 (Samsung) with 6 (LG), 3 (Doosan) with 7 (Nexen), and 4 (Lotte) with 8 (Hanwha)].

4. Among all 532 games, the game fixture for the first 500 games are fixed before the season starts (see Table IV]. First week has 8 games, and from the second week 24 games are held every week. The third week of July (the 16th week of the season) is another exception and regular season matches are held only for the first half of the week. On Saturday (July 21) there will be a special event so-called "All Star Game". Accordingly, after 22 weeks, all 500 [= $8($ the 1 st week $)+12$ (the 3 rd week of July $)+24 \cdot 20$ (regular weeks)] games are played, and remaining 32 games start from September 2. Fixture of those remaining 32 games will be determined during the season, reflecting the unavoidable changes made by e.g., canceled game due to heavy rain.
5. Around the Children's' Day in Korea (May 5), the most popular day for kids to watch a baseball game in a stadium with family, four stadiums for three consecutive games are chosen as the homes of Samsung, SK, Kia, and LG. Every second year, the four teams alternate so that Doosan, Nexen, Lotte, and Hanwha will be the ones in the 2013 season.

6. No team is allowed to play more than 6 home games consecutively.

7. No team is allowed to play more than 6 away games consecutively, with one exception: KBO noticed the longer travel distance of Lotte, and allows the team to play 9 away games in a row, before the 500 th game of the season (from June 15 to 24 in the 2012 season). In Table IV] we underline those 9 games in the 11th and 12th weeks of the schedule.

The above scheduling rules set by KBO show that the organization is trying to provide equal bases for all the teams. Especially, the rule 7 makes Lotte an exception to compensate the team's longer distance as shown in Tables [I and III, and Fig. 2. We use the KBO schedule for the 2012 season in Table IV to compute the total traveling distance and time of the season. For example, the team SK plays three home games at Munhak stadium in the 1st week $\left(\sum d=0\right)$, and travels to Mokdong stadium to play three away games with $\mathrm{NX}\left(\sum d=d_{28}\right)$, and then 

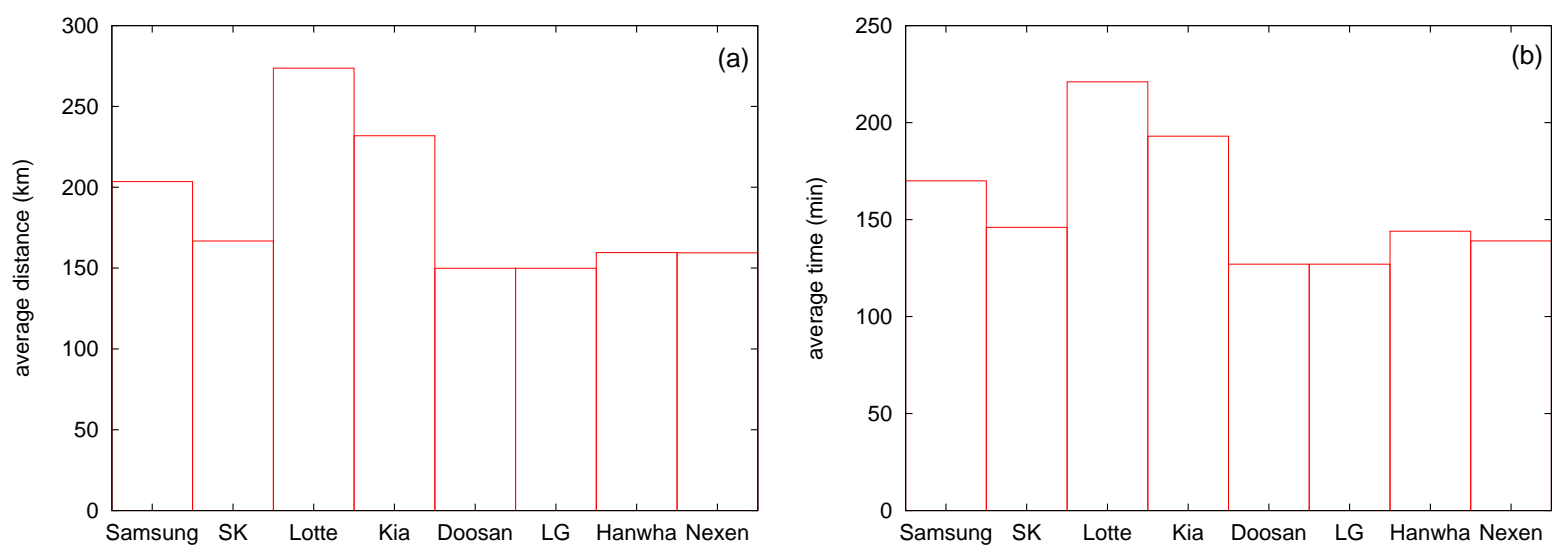

FIG. 2. (Color online) Average (a) distance $\left(\equiv\left(d_{i} \bullet+d_{\bullet}\right) / 2\right)$ and (b) time $\left(\equiv\left(t_{i} \bullet+t_{\bullet}\right) / 2\right)$ from Table II and III Clearly shown is that the team Lotte (see Fig. 1) has larger average distance and time to travel than other teams.

TABLE IV. Baseball schedule for 2012 season by KBO. Fixture of all 500 games is given. For acronyms of team names and stadiums, see Table I. Each pair of two teams in the form XX/YY in the table denotes three consecutive games, one per day, with XX (YY) the home (away) team. See text for the rules set by KBO. To compensate the longer travel distance of Lotte, KBO allows the team to have 9 consecutive away games, as underlined in the schedule (in the 11th and the 12th weeks).

\begin{tabular}{|c|c|c|c|c|c|c|c|c|c|c|c|c|c|c|}
\hline & \multicolumn{7}{|c|}{ Tuesday - Thursday } & \multicolumn{7}{|c|}{ Friday - Sunday } \\
\hline Week & Jamsil & Mokdong & Munhak & Daegu & Gwangju & Daejeon & Sajik & Jamsil & Mokdong & Munhak & Daegu & Gwangju & Daejeon & Sajik \\
\hline 1 & \multicolumn{7}{|c|}{ No game } & $\mathrm{DS} / \mathrm{NX}^{\mathrm{a}}$ & - & $\mathrm{SK} / \mathrm{KA}^{\mathrm{a}}$ & $\mathrm{SS} / \mathrm{LG}^{\mathrm{a}}$ & - & - & $\mathbf{L T} / \mathrm{HH}^{\mathrm{a}}$ \\
\hline 2 & LG/LT & NX/SK & - & - & $\mathrm{KA} / \mathrm{SS}$ & $\mathrm{HH} / \mathrm{DS}$ & - & LG/KA & - & $\mathrm{SK} / \mathrm{HH}$ & SS/NX & - & - & $\mathrm{LT} / \mathrm{DS}$ \\
\hline 4 & LG/NX & - & SK/DS & SS/LT & $\mathrm{KA} / \mathrm{HH}$ & - & - & $\mathrm{DS} / \mathrm{KA}$ & - & $\mathrm{SK} / \mathrm{SS}$ & - & - & $\mathrm{HH} / \mathrm{NX}$ & $\mathrm{LT} / \mathrm{LG}$ \\
\hline 5 & $\mathrm{LG} / \mathrm{HH}$ & NX/LT & - & SS/DS & $\mathrm{KA} / \mathrm{SK}$ & - & - & $\mathrm{LG} / \mathrm{DS}^{\mathrm{b}}$ & - & SK/LT ${ }^{b}$ & $\mathbf{S S} / \mathbf{H H}^{\mathrm{b}}$ & $\mathrm{KA} / \mathrm{NX}^{\mathrm{b}}$ & - & - \\
\hline 6 & $\mathrm{DS} / \mathrm{SK}$ & NX/LG & - & - & - & $\mathrm{HH} / \mathrm{KA}$ & LT/SS & LG/SS & - & SK/NX & - & $\mathrm{KA} / \mathrm{DS}$ & $\mathrm{HH} / \mathrm{LT}$ & - \\
\hline 8 & LG/NX & - & SK/DS & SS/LT & $\mathrm{KA} / \mathrm{HH}$ & - & - & $\mathrm{DS} / \mathrm{LT}$ & $\mathrm{NX} / \mathrm{HH}$ & - & SS/SK & $\mathrm{KA} / \mathrm{LG}$ & - & - \\
\hline 9 & DS/KA & NX/SK & - & - & - & $\mathrm{HH} / \mathrm{SS}$ & LT/LG & LG/HH & - & SK/KA & SS/DS & - & - & LT/NX \\
\hline 10 & DS/SK & $\mathrm{NX} / \mathrm{LG}$ & - & - & KA/SS & $\mathrm{HH} / \mathrm{LT}$ & - & LG/DS & - & $\mathrm{SK} / \mathrm{SS}$ & - & - & $\mathrm{HH} / \mathrm{NX}$ & $\mathrm{LT} / \mathrm{KA}$ \\
\hline 11 & LG/SK & - & - & $\mathrm{SS} / \mathrm{HH}$ & $\mathrm{KA} / \mathrm{NX}$ & - & $\mathrm{LT} / \mathrm{DS}$ & $\mathrm{DS} / \mathrm{SS}$ & $\underline{\mathrm{NX} / \mathrm{LT}^{\mathrm{c}}}$ & $\mathrm{SK} / \mathrm{HH}$ & - & $\mathrm{KA} / \mathrm{LG}$ & - & - \\
\hline 12 & $\mathrm{DS} / \mathrm{NX}$ & - & $\mathrm{SK} / \mathrm{LT}^{\mathrm{c}}$ & $\mathrm{SS} / \mathrm{KA}$ & - & $\mathrm{HH} / \mathrm{LG}$ & - & $\underline{\mathrm{LG} / \mathrm{LT}^{\mathrm{c}}}$ & $\mathrm{NX} / \mathrm{SS}$ & - & - & $\mathrm{KA} / \mathrm{SK}$ & $\mathrm{HH} / \mathrm{DS}$ & - \\
\hline 13 & LG/KA & NX/DS & - & $\mathrm{SS} / \mathrm{SK}$ & - & - & $\mathrm{LT} / \mathrm{HH}$ & $\mathrm{DS} / \mathrm{LT}$ & - & $\mathrm{SK} / \mathrm{LG}$ & $\mathrm{SS} / \mathrm{NX}$ & - & $\mathrm{HH} / \mathrm{KA}$ & - \\
\hline 18 & $\mathrm{LG} / \mathrm{HH}$ & - & $\mathrm{SK} / \mathrm{NX}$ & $\mathrm{SS} / \mathrm{DS}$ & - & - & $\mathrm{LT} / \mathrm{KA}$ & $\mathrm{DS} / \mathrm{KA}$ & NX/LG & - & - & - & $\mathrm{HH} / \mathrm{SK}$ & $\mathrm{LT} / \mathrm{SS}$ \\
\hline 19 & LG/LT & - & SK/SS & - & $\mathrm{KA} / \mathrm{NX}$ & $\mathrm{HH} / \mathrm{DS}$ & - & DS/SK & $\mathrm{NX} / \mathrm{HH}$ & - & SS/LG & $\mathrm{KA} / \mathrm{LT}$ & - & - \\
\hline 20 & LG/KA & NX/DS & - & $\mathrm{SS} / \mathrm{HH}$ & - & - & $\mathrm{LT} / \mathrm{SK}$ & $\mathrm{DS} / \mathrm{SS}$ & - & $\mathrm{SK} / \mathrm{KA}$ & - & - & $\mathrm{HH} / \mathrm{LG}$ & $\mathrm{LT} / \mathrm{NX}$ \\
\hline 21 & $\mathrm{DS} / \mathrm{NX}$ & - & $\mathrm{SK} / \mathrm{HH}$ & SS/LT & $\mathrm{KA} / \mathrm{LG}$ & - & - & LG/SS & NX/SK & - & - & - & $\mathrm{HH} / \mathrm{KA}$ & $\mathrm{LT} / \mathrm{DS}$ \\
\hline 22 & $\mathrm{DS} / \mathrm{LG}$ & - & SK/LT & - & $\mathrm{KA} / \mathrm{SS}$ & $\mathrm{HH} / \mathrm{NX}$ & - & $\mathrm{DS} / \mathrm{HH}$ & - & - & $\mathrm{SS} / \mathrm{NX}$ & $\mathrm{KA} / \mathrm{SK}$ & - & $\mathrm{LT} / \mathrm{LG}$ \\
\hline
\end{tabular}

a The schedule of the opening week is fixed.

b Around the Children's Day, home stadiums are fixed.

c Nine consecutive away games allowed for Lotte.

back to Munhak stadium for three home games with $\mathrm{HH}$ $\left(\sum d=d_{28}+d_{82}\right)$ in the 2 nd week. SK keeps traveling following the schedule in Table IV so that its total traveling distance is $\sum d=d_{28}+d_{82}+d_{23}+d_{35}+d_{52}+\cdots+d_{24}$. When there are consecutive away games before and after Monday, we assume that the team travels directly from one place to other, without having a break on Monday at the team's home. We also compute the total travel- ing time similarly to the total traveling distance, and list the both in Table $\nabla$. Clearly seen in Table $\nabla$ is that the above KBO rules do not successfully reduce the unequal distribution of traveling distances and times. For example, even though Lotte is given an exception (the rule 7) the team travels much longer distance $(66 \%)$ than LG. Another interesting observation is that although Doosan and LG are located at the same home position, the travel 
TABLE V. Total traveling distance and time for the 2012 schedule in Table IV.

\begin{tabular}{c|c|c|c}
\hline \hline$\#$ & team & distance $(\mathrm{km})$ & time $(\mathrm{min})$ \\
\hline 1 & Samsung & 9086.9 & 7484 \\
2 & SK & 6714.7 & 5881 \\
3 & Lotte & 9204.9 & 7594 \\
4 & Kia & 8311.1 & 7007 \\
5 & Doosan & 6795.0 & 5692 \\
6 & LG & 5538.0 & 4764 \\
7 & Hanwha & 7017.0 & 6154 \\
8 & Nexen & 6552.4 & 5645 \\
\hline & average & 7402.5 & 6278 \\
\hline \hline
\end{tabular}

distances are quite different: Doosan travels 23\% longer distance compared to LG.

\section{FORMULATION}

The total traveling distance of the team $i$ is given by

$$
d_{i}=\sum_{(k, l)} d_{k l}
$$

where the sum is over the traveling path for a given schedule, and $d_{k l}$ is the distance from $k$ to $l$ in Table II Differently from the usual TTP in which the objective function to minimize is $\sum_{i} d_{i}$, we in this work propose to minimize the unfairness of the schedule, measured by the standard deviation of $d_{i}$. In analogy to the statistical physics problem finding the ground state of a system, we call it as "energy" $E$ of the system, although the term does not mean much in the context of baseball game scheduling. Our objective function to minimize in the present work is given by

$$
E=\sqrt{\frac{1}{8} \sum_{i=1}^{8}\left(d_{i}-m\right)^{2}}=\sqrt{\left\langle d_{i}^{2}\right\rangle-\left\langle d_{i}\right\rangle^{2}} \equiv \sigma_{d}
$$

where the mean $m \equiv\left\langle d_{i}\right\rangle \equiv(1 / 8) \sum_{i=1}^{8} d_{i}$, and $\left\langle d_{i}^{2}\right\rangle \equiv$ $(1 / 8) \sum_{i=1}^{8} d_{i}^{2}$. In contrast to the original TTP to minimize $m$, our purpose here is to minimize $E$. In the ideal situation of $E=0$, all teams travel equal total distances, while the larger is $E$, the more unfair the schedule becomes. We also use the standard deviation $\sigma_{t}$ of the travel time $t_{i} \equiv \sum_{(k, l)} t_{k l}$ with $t_{k l}$ being the travel time from $k$ to $l$ in Table III, to define the energy function $E$.

In statistical physics, finding of the ground state configuration of a model system is the one of the most welldeveloped topics. The standard way to tackle the issue is to set up an energy function of the given model system and minimize the energy in a systematic way. In this regard, the most standard methodology is to use the Monte-Carlo (MC) simulation technique [14]. The standard Metropolis algorithm for local update is adopted:
The local MC try with the energy difference $\Delta E$ is accepted if $\Delta E \leq 0$. Otherwise (i.e., if $\Delta E>0$ ), the MC try is accepted at the probability of $e^{-\Delta E / T}$ with the temperature $T$. The Metropolis MC method is then combined with the simulated annealing technique. In more detail, we start from a high temperature, and decrease the temperature slowly until the zero temperature is reached. The similar MC method with the simulated annealing technique has been applied for the TSP [2]. In Ref. 3, a microcanonical MC method combined with an annealing technique called the entropic annealing method has also been applied for the TSP. In the present study, we do not aim at developing a more advanced methodology in finding the fairest tournament schedule, but we simply apply the well-known simulated annealing MC method to tackle the problem. Accordingly, we do not claim that we have found the fairest schedule of the KBL, but rather like to emphasize that a simple application of the well-known methodology in statistical physics can significantly increase the fairness of the schedule of the KBL.

The other important issue to discuss is what are the local update rules allowed in the MC simulation. In Ref. 10, all five different ways of update are proposed: SwapHomes, SwapRounds, SwapTeams, PartialSwapRounds, and PartialSwapTeams. It has been shown that the first three swapping methods are not sufficiently efficient to explore the entire configuration space [10, 11]. In this work, we only use SwapRounds and SwapHomes for updates due to their simplicity: In the former we pick two different rounds, e.g., Fri-Sun of the week 7 and Tue-Thu of the week 6 , and swap the two entirely. In the SwapHomes update, we pick two pairs, e.g., NX/SK and SK/NX at two different rounds Tue-Thu of the week 2 and Fri-Sun of the week 6 , and then swap the two. These two update methods (SwapRounds and SwapHomes) are the simplest ones among the five in Ref. 10 and it is clear that the two update methods preserve the KBO rules explained in Sec II if the schedule in Table IV is used as an initial configuration of MC simulations.

\section{RESULTS}

We start from a sufficiently high temperature $T=100$ with the KBO schedule in Table [V] as an initial condition and decrease $T$ slowly with the temperature step $\Delta T=1.0$ until $T=0$ is arrived. For 22 weeks of the season in Table IV] there are all 42 rounds, with two rounds per week except for the 1st and 16th weeks. At each temperature, we perform 10,000 MC steps, with one MC step composed of one SwapRounds update and one SwapHomes updates per round. Our update rules do not change the number of home games and away games for each team, and thus the rule 1 of KBO in Sec. II is automatically obeyed. The schedule of the first week is fixed (rule 3) in our simulation, and the "All Star Game" in the 16 th week is also fixed (see rule 4). No teams are allowed 

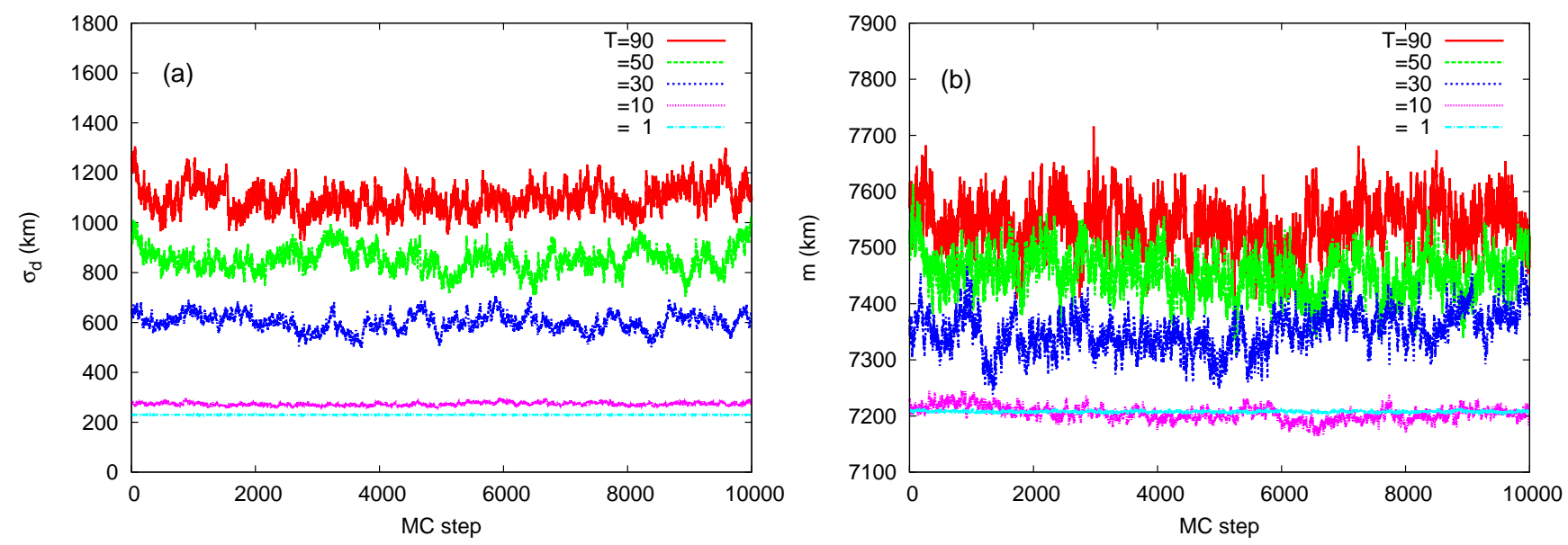

FIG. 3. (Color online) Evolution of (a) the standard deviation of traveling distance $\sigma_{d}$ and (b) the average traveling distance $m$ versus Monte-Carlo time step. We start from a high enough temperature $T=100$ and decrease $T$ slowly after 10,000 MC updates at each temperature. As $T$ is lowered from $T=90$, to 50, 30, 10, and 1 (from top to bottom curves), $\sigma_{d}$ systematically decreases. (b) Although not explicitly intended $m$ is also found to decrease with $T$ for $T \gtrsim 10$.

TABLE VI. Optimized schedule in this work with maximum fairness in traveling distances.

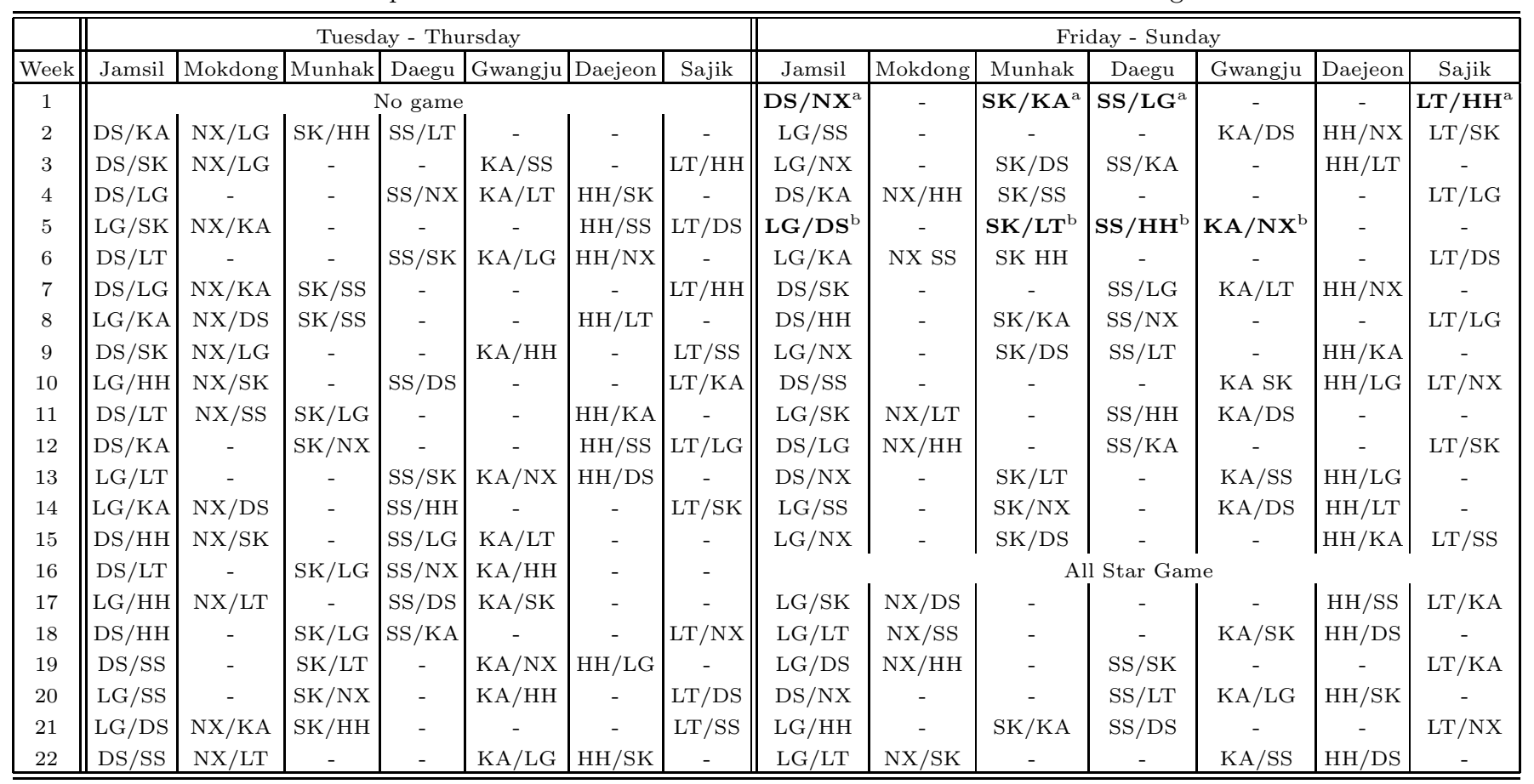

a The schedule of the opening week is fixed.

b Around the Children's Day, home stadiums are fixed.

to have more than six consecutive home games (rule 6), nor six consecutive away games (rule 7). The exception made for Lotte (rule 7 in Sec. III) is not necessary in our simulation. The rule 5 on the home stadiums around the Children's Day is also obeyed during simulations. Consequently, all rules (except the unnecessary exception for Lotte in rule 7) are equally obeyed for all teams during our simulations at any temperature, and the ground state as an outcome of the simulated annealing MC simu- lations gives us the tournament schedule that is expected to be as fair as possible in terms of the travel distance (for $\left.E=\sigma_{d}\right)$ or the travel time $\left(E=\sigma_{t}\right)$. We repeat the same annealing procedure 10 times for the fairness of traveling distances $\left(E=\sigma_{d}\right)$ and once for the fairness of traveling times $\left(E=\sigma_{t}\right)$. The results are almost the same for $E=\sigma_{d}$ and $E=\sigma_{t}$, and we below mostly present our results for the former.

In Fig. 3, we show the evolution of (a) $\sigma_{d}$ and (b) $m$ in 
TABLE VII. Total traveling distance of each team is listed for the optimized schedule in Table VI obtained from MC simulated annealing with the fairness of traveling distances as the objective function. The total traveling times obtained when the standard deviation of the traveling times is chosen as the energy function are also listed. Note that the average distance and time are smaller than the ones in actual KBO schedule in Table V] See text for more details.

\begin{tabular}{c|c|c|c}
\hline \hline$\#$ & team & distance $(\mathrm{km})$ & time $(\mathrm{min})$ \\
\hline 1 & Samsung & 7185.6 & 6103 \\
2 & SK & 7163.5 & 6071 \\
3 & Lotte & 7252.7 & 6233 \\
4 & Kia & 7205.8 & 6139 \\
5 & Doosan & 6980.0 & 5986 \\
6 & LG & 6866.3 & 5852 \\
7 & Hanwha & 6898.5 & 6064 \\
8 & Nexen & 7183.4 & 6074 \\
\hline & average & 7092.0 & 6065 \\
\hline \hline
\end{tabular}

Eq. (2) during MC simulated annealing. As the temperature is lowered, it is shown that our simulation successfully lowers the energy, or increase fairness in the travel distance. Although our simulation tries to minimize $\sigma_{d}$, $m$ is also found to decrease as a byproduct of annealing as shown in Fig. 3(b). Ten independent simulated annealing with $E=\sigma_{d}$ give us ten ground state configurations, and the one with the lowest energy has the configuration tabulated in Table VI. We emphasize that our optimized baseball schedule keeps all the scheduling constraints by $\mathrm{KBO}$, but with significantly enhanced fairness in travel distances.

In Table VII, we list the total traveling distances of teams for the ground state configuration in Table VI In comparison to the corresponding values for the actual schedule run by KBO in Table $\mathrm{V}$, our optimized schedule clearly has more even distribution of travel distances. In Table VII, we also include the result from our MC simulated annealing with $E=\sigma_{t}$, i.e., the fairness in traveling times as the objective function. The traveling time of each team has much smaller variance in comparison to the original schedule by KBO.

To summarize our main findings, we plot in Fig. 4 the traveling distances for (a) the KBO schedule in Tables IV and $\mathrm{V}$ and (b) our optimized schedule in Tables VI and VII Clearly revealed is that our optimization scheme results in much better fairness in travel distances. The corresponding plot for the total traveling time looks very similar to Fig. 团and thus not included in the present paper. Although the team Lotte still has the longest distance to travel, the difference to LG is now only about $6 \%$ (compared to $66 \%$ in original KBO schedule). Also, the difference between LG and Doosan shrinks to $2 \%$ (compared to $23 \%$ in $\mathrm{KBO}$ schedule). We also report somehow unexpected result of our scheme: Although not intended, the total sum of all the traveling distances of eight teams in our optimized schedule has smaller value than the one for the original KBO schedule. Accordingly, we conclude that we manage to find better schedule in terms of both fairness and effectiveness, in comparison to the KBO schedule.

\section{SUMMARY AND DISCUSSION}

We have studied numerically the traveling tournament problem in Korean Baseball League. Instead of using the total sum of traveling distances as the objective function to minimize, we focus on the fairness in the traveling distances of baseball teams. More specifically, we use the standard deviation of the traveling distance as the energy function in statistical mechanics, and apply the simulated annealing Monte-Carlo method to find the ground state configuration. The resulting timetable for the baseball games for year 2012 is shown to be better than the schedule posted by the Korean Baseball Organization, in terms of both the fairness, and the effectiveness. In other words, our optimized schedule yields more equal traveling distances across teams, and the total sum of total traveling distances is shorter than the corresponding value of the posted schedule by the organization.

We admit that there could be some other implicitly posed constraints not reflected in our simulations. However, if those constraints are explicitly revealed to public, our methodology can be applied with little changes in the MC program. We strongly believe that statistical physics approach like presented in this work has a broad applicability in a broad range of problems we encounter everyday, and hope to get encountered by more interesting problems in the real world of society.

\section{ACKNOWLEDGMENTS}

This work was done as a team project in the 9th KIASAPCTP Winter School on Statistical Physics in Korea (2012). B.J.K. was supported by the National Research Foundation of Korea (NRF) grant funded by the Korea government (MEST) (No. 2011-0015731).
[1] G. Gutin and A. Punnen, The traveling salesman problem and its variations, Vol. 12 (Kluwer Academic Pub, 2002).

[2] S. Kirkpatrick, C. Gelatt Jr, and M. Vecchi, Science 220,
671 (1983)

[3] J. Lee and M. Y. Choi, Phys. Rev. E 50, R651 (1994).

[4] R. Brady, Nature 317, 804 (1985). 

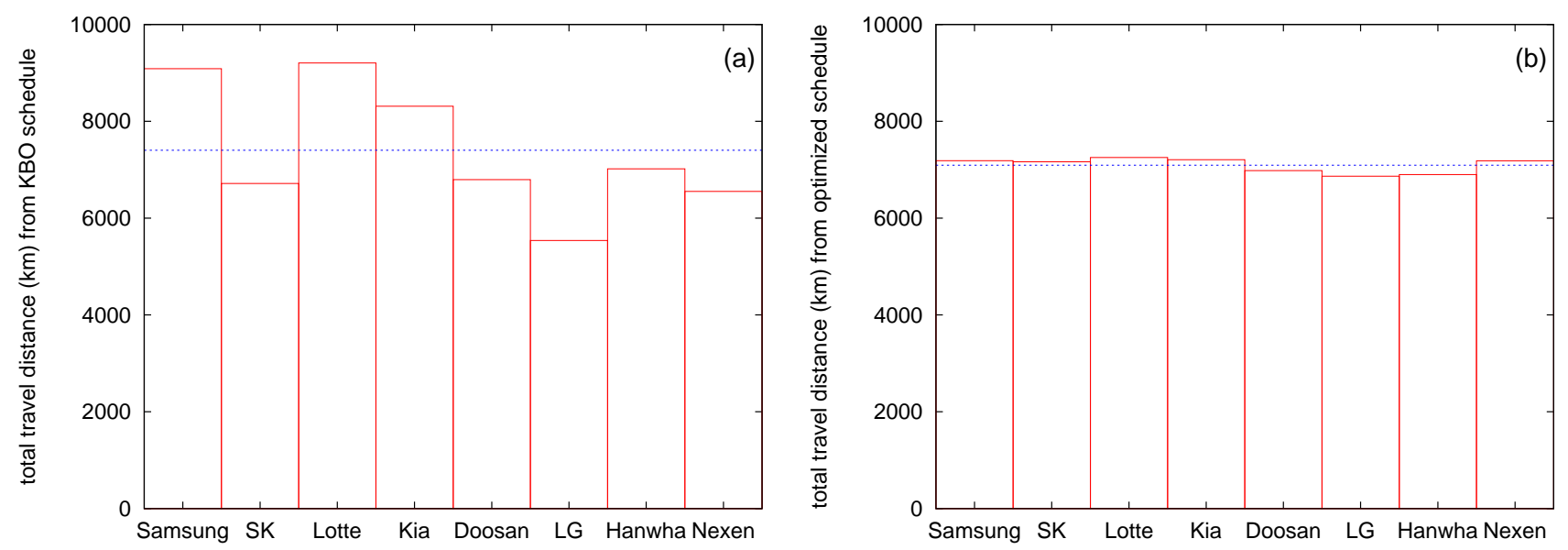

FIG. 4. (Color online) (a) Total traveling distance for the KBO 2012 schedule in Table IV Total traveling distance is quite different for each team, with Lotte the worst and LG the best. (b) Total traveling distance from the optimized schedule in Table VI] Our optimized schedule provides much better fairness in view of the distribution of traveling distances. The horizontal dotted lines in (a) and (b) are average total travel distance over eight teams. Interestingly, the average distance is shorter for the optimized schedule than the one in the actual KBO schedule, which means our suggested schedule is better than the KBO schedule in terms of both fairness and effectiveness.

[5] M. Dorigo and T. Stüzle, Ant colony optimization (Bradford Books, 2004).

[6] M. Dorigo and L. Gambardella, IEEE Transactions on Evolutionary Computation 1, 53 (1997).

[7] D. Briskorn, Sports leagues scheduling: Models, combinatorial properties, and optimization algorithms, 603 (Springer Verlag, 2008).

[8] G. Kendall, S. Knust, C. Ribeiro, and S. Urrutia, Computers \& Operations Research 37, 1 (2010).

[9] K. Easton, G. Nemhauser, and M. Trick, in Principles and Practice of Constraint Programming -CP 2001 (Springer, 2001) pp. 580-584.

[10] A. Anagnostopoulos, L. Michel, P. Hentenryck, and Y. Vergados, Journal of Scheduling 9, 177 (2006).
[11] P. Chen, G. Kendall, and G. Berghe, in Computational Intelligence in Scheduling, 200\%. SCIS'0\%. IEEE Symposium on (IEEE, 2007) pp. 19-26.

[12] M. Trick, Practice and Theory of Automated Timetabling III , 242 (2001); A. Schaerf, Constraints 4, 43 (1999).

[13] J. Rawls, A Theory of Justice (Oxford University Press, 1999); R. Dworkin, Sovereign Virtue: The Theory and Practice of Equality (Harvard University Press, 2002); S. K. Baek, J.-K. Choi, and B. J. Kim, "Dworkin's paradox," Unpublished.

[14] M. Newman and G. Barkema, Monte Carlo Methods in Statistical Physics (Clarendon Press, 1999). 